\title{
Performing Service Composition in Opportunistic Networks
}

\author{
Fadhlallah Baklouti \\ IRISA, Université Bretagne Sud \\ Vannes, France \\ fadhlallah.baklouti@univ-ubs.fr
}

\author{
Nicolas Le Sommer \\ IRISA, Université Bretagne Sud \\ Vannes, France \\ nicolas.le-sommer@univ-ubs.fr
}

\author{
Yves Mahéo \\ IRISA, Université Bretagne Sud \\ Vannes, France \\ yves.maheo@univ-ubs.fr
}

\begin{abstract}
Networks are more and more composed of heterogeneous devices that are intermittently connected. Intermittences are induced by the mobility of devices communicating through short-range wireless interfaces, and by the sleep phases made by devices for energy saving purposes. Discovering, invoking and compositing services in such networks can therefore be difficult tasks. This paper presents a middleware system to achieve these tasks efficiently, thanks to opportunistic networking and computing techniques that are designed to minimize the execution time of these tasks, as well as to maximize their success ratio.

Index Terms-Opportunistic software systems, service composition
\end{abstract}

\section{INTRODUCTION}

Nowadays, wireless networks are composed of a variety of heterogeneous devices that can communicate directly with one another, ranging from resource-constrained sensors to mobile devices and smart home appliances. Applications relying on these kind of networks are generally distributed over a collection of devices, because devices often have limited processing, memory, storage, communication and battery resources. Progress in mastering device-to-device communication (D2D) has been possible as techniques, such as opportunistic networking or delay/disruption-tolerant networking, emerged to cope with the frequent and unpredictable connectivity disruptions, and with the additional delays introduced in D2D data exchanges, both resulting from the mobility of devices that communicate using short radio range network interfaces, and from the periods of sleep mode implemented by devices for energy saving purposes. Building distributed applications as compositions of services is a usual software development approach, which can become a challenging task in opportunistic and delay/disruption-tolerant networks. Indeed, composition strategies, dedicated to conventional Internet contexts, are likely to cause prohibitive delays, and to suffer from transmission failures. In Internet, these transmissions are more or less considered always possible and efficient. In opportunistic networks, this assumption no longer holds. Indeed, service composition multiplies the constraints because fulfilling a composite service request involves the invocation of multiple services, and any of these services being susceptible to be out of reach at any moment.

Composition of services in opportunistic networks has been studied in a limited number of works [1], [2], [3], [4] so far. These works define solutions for the parallel execution of compositions [4], the recovery of partial compositions [5] and the selection of service providers according to metrics such as the shortest temporal distance, which is the minimum time needed to send data from one node to another one, and the service load, which reflects the workload of a given service [1]. Nevertheless, they do not investigate and compare the performances provided by the two well known composition strategies (i.e. the orchestration and the choreography) in different type of opportunistic networks, or against different criteria for the selection of service providers.

In this paper, we present a middleware system that supports the discovery, the selection, the invocation and the composition of services in opportunistic networks. This system has been developed using the opportunistic communication framework C3PO [6]. It implements the choreography and the orchestration composition strategies. These strategies can be parameterized with a utility function that makes it possible to estimate the delay and the success of a transmission between a service client and a service provider, and to select the providers according to these values. Two implementations of the utility function are considered and evaluated in this paper. The first one is based on location, and the second one is based on the reception times of the service advertisements emitted by service providers. In this work, we assume on one hand that each device in the network can play the role both of service provider and of service client, and on the other hand, that a same service may be offered by several providers in the network. In this paper, we also compare the success ratio and the execution time provided by the composition strategies and the utility functions in two different scenarios. The first scenario involves people moving around in an open area, and the second scenario involves people attending a race and moving along a predetermined running path.

The rest of this paper is organized as follows. Section II details and formalizes the processes of discovery, selection and composition of the services. Section III presents the evaluation results we obtained for our system on the two above-mentioned scenarios. Section IV summarizes our contribution.

\section{LOCATION-BASED VS TIME-BASED SERVICE COMPOSITION}

The composition system we have implemented selects, for each composition request, the most efficient and reliable composition strategy (i.e. the choreography or the orchestration) that must be applied according to two criteria of quality of 
service, namely the success ratio of the composition request and its execution time. These two criteria are transposed to the selection of service providers that must be enrolled in a composition process. Providers are thus selected according to an estimation of the time needed to reach them, and to an estimation of the ratio of successful transmissions. These estimations are computed by a utility function. In the rest of this section, we present two implementations of this function, and how they are used.

\section{A. Selection of providers}

Service providers are selected by the utility function $\varphi$ defined as follows:

$$
\left\{\begin{array}{l}
\varphi\left(S_{i}\right)=P_{j}, f\left(p_{j}\right)=\max \left(f\left(P_{l}\right)\right), P_{l} \in R\left[S_{i}\right] \\
f(x)=\frac{\mu}{t(x)}+(1-\mu) \times s(x)
\end{array}\right.
$$

In this formula, $P_{j}$ is the provider having the highest value, calculated by function $f$, among the providers that offer service $S_{i}$ (identified by $R\left[S_{i}\right]$, where $R$ stands for the local registry). Function $f$ computes a value based on the estimation of the time needed to reach a provider and on the estimation to join that provider successfully. These estimations are respectively noted $t(x)$ and $s(x)$ for a provider $x$. Parameter $\mu$ makes it possible to promote one quality of service parameter to the detriment of the other. $t(x)$ and $s(x)$ are computed either based on temporal or geographical information. Hereafter, we show how these values are calculated.

a) Time-based implementation of the utility function: The time needed to send a service request to a provider is estimated from the average of the transmission delays of the service advertisements broadcast by the provider. The success ratio of a transmission is the proportion of advertisements received by the local host among the advertisements emitted by a provider. To estimate this ratio, we assume that all devices send service advertisements with the same period of time (noted $\delta$ in the formulas hereafter). The time-based utility function we have defined operates on a sliding window of $k$ values (i.e., we only consider the last $k$ advertisements that are received by the local host). The estimated time $t$ and the success ratio $s$ for provider $P$ are therefore formally defined by:

$$
\begin{aligned}
t(P) & =\frac{\sum_{i=1}^{k}\left(\beta_{i}^{P}-\alpha_{i}^{P}\right)}{k} \\
s(P) & =\frac{k}{1+\left\lfloor\sum_{i=2}^{k}\left(\left(\beta_{i}^{P}-\beta_{i-1}^{P}\right) / \delta\right)\right\rfloor}=\frac{k}{1+\left\lfloor\left(\left(\beta_{k}^{P}-\beta_{1}^{P}\right) / \delta\right)\right\rfloor}
\end{aligned}
$$

where $\alpha_{i}^{p}$ and $\beta_{i}^{p}$ are respectively the emission time and the reception time of the $i$-th advertisement received from provider $P$. By computing an average of the transmission delays instead of considering only the last one, we promote, to the detriment of the providers that are met in fleeting way, those that are reached the most frequently with a minimum of delay either directly or via intermediate devices.

b) Location-based implementation of the utility function: When sending an advertisement, the composition system includes in this advertisement the location of the local host. Doing so, the devices receiving this advertisement can compare their own location with that of the provider. Similarly to the previous utility function, this function operates on the last $k$ advertisements received from a given provider. This function computes an estimation of the transmission delay considering the average of the distances between a provider and the local host. By computing the average of the distances between the local host and a provider instead of considering only the last distance between them, we promote the providers that are closest to the local host during a given period of time instead of those that are briefly close. The estimation of the time needed to reach service provider $P$ is equal to the reception delay of the advertisement $i$, such that the distance $d_{i}^{P}$ between the local host and the provider is the closest to the average of the distances traveled by the $k$ advertisements received by the local host:

$$
t(P)=\left(\beta_{i}^{P}-\alpha_{i}^{P}\right), d_{i}^{P} \approx \frac{\sum_{l=1}^{k} d_{l}}{k}, i \in[1, k]
$$

To estimate the success ratio of a transmission between a client and a provider, we consider the $k$ last advertisements received by a (local) client from a provider, and we compute the average of the distances between them based on their respective location at the emission time and at the reception time. The probability of reaching a provider usually decreases when the distance between the local device and the provider increases. That is why we have defined the success estimation function as a multiplicative inverse function, shifted to the left by -1 , which takes $d^{P}$ as a parameter. This function returns a value close to 1 when $d^{P}$ is small and a value close to 0 when $d^{P}$ is big. It is defined as follow:

$$
s(p)=\frac{1}{1+d^{p}}, d^{P}=\frac{\sum_{j=1}^{k} d_{j}^{p}}{k}
$$

\section{B. Service Composition}

Hereafter, we present both the estimations of the execution time and of the success ratio of a composition for the two strategies implemented in our composition system. For that, let us consider a composition $C$ of $n$ services identified respectively by $S_{i}, i \in[1, n]$, a set of $m$ providers that offer one or several services. Let us also consider that composition $C$ is emitted by a requester $\Lambda$.

1) Estimation of the composition time: The composition time of $C$ is defined for the orchestration-based strategy by:

$$
\tau(C)=\sum_{i=1}^{n} 2 * t_{\Lambda}\left(P_{l}\right), P_{l}=\varphi_{\Lambda}\left(S_{i}\right)
$$

where $P_{l}$ is the provider of the service $S_{i}$ that has been selected by the utility function $\varphi$. In the orchestration-based strategy, the response must be returned to the device $\Lambda$ that has initiated the composition request. Thus, the time $t_{\Lambda}\left(P_{l}\right)$ is multiplied by 2 to consider this round trip.

Concerning the choreography-based strategy, the estimation of the composition time is defined by:

$$
\left\{\begin{array}{l}
\tau(C)=t_{\Lambda}\left(P_{1}\right)+\sum_{i=1}^{n-1} t_{P_{i}}\left(P_{i+1}\right)+t_{P_{n}}(\Lambda) \\
P_{1}=\varphi_{\Lambda}\left(S_{1}\right) \\
P_{i+1}=\varphi_{P_{i}}\left(S_{i+1}\right)
\end{array}\right.
$$

where $t_{\Lambda}\left(P_{1}\right)$ is the estimation of the time needed to send the composition request from the requester $\Lambda$ to the first 
provider, $t_{P_{n}}(\Lambda)$ is the estimation of the time needed to send the composition result from provider $P_{n}$ of the last service $S_{n}$ to the composition requester $\Lambda$. The rest of the formula $\tau(C)$ is the sum of the estimations of intermediate composition times, where $t_{P_{i}}\left(P_{i+1}\right)$ is the estimation of the time needed to send the composition request from provider $P_{i}$ to the next provider $P_{i+1}$.

2) Estimation of success ratio: As mentioned in the section related to the estimation of the composition time, in the orchestration-based strategy, the response of each service invocation is returned to the requester $\Lambda$. Thus, the estimation of the success of an orchestration-based composition is defined as the product of the square of the transmission success estimation of a request from the requester $\Lambda$ to the providers enrolled in the composition process. This estimation is defined by:

$$
\gamma(C)=\prod_{i=1}^{n} s_{\Lambda}\left(P_{l}\right)^{2}, P_{l}=\varphi_{\Lambda}\left(S_{i}\right)
$$

Regarding the choreography-based strategy, the estimation of the success of a composition is defined as the product of the transmission success estimation of a request from the requester $\Lambda$ to the first provider $\left(P_{1}\right)$ with the transmission success estimation of the composition result from the last provider $\left(P_{n}\right)$ to the requester $\Lambda$, and with the intermediate transmission success estimations.

$$
\left\{\begin{array}{l}
\gamma(C)=s_{\Lambda}\left(P_{1}\right) \times s_{P_{n}}(\Lambda) \times \prod_{i=1}^{n-1} s_{P_{i}}\left(P_{i+1}\right) \\
P_{1}=\varphi_{\Lambda}\left(S_{1}\right) \\
P_{n}=\varphi_{P_{n-1}}\left(S_{n}\right) \\
P_{i+1}=\varphi_{P_{i}}\left(S_{i+1}\right)
\end{array}\right.
$$

\section{RESUlts AND EVAlUATIONS}

\section{A. Evaluation setup}

We have conducted a series of emulations to evaluate the composition success ratio and the composition time provided respectively by the orchestration strategy and the choreography strategy configured successively with the time-based utility function and the location-based utility function. These evaluations were conducted using the LEPTON emulator ${ }^{1}$, which allows us to run the same code as the one we deploy on Android mobile devices. Two different scenarios have been considered during these evaluations, namely an open area of $500 \times 500 \mathrm{~m}$ in which 200 people move around according to the Levy Walk mobility model, and a part of the city of Vannes in France where a group of 200 people attend to a running event and move along the predetermined path. In these scenarios, people move at a speed between 0.5 and $2 \mathrm{~m} / \mathrm{s}$, while communicating using smartphones equipped with WiFi Direct. The maximum communication range of the devices is $80 \mathrm{~m}$. Each device provides 5 services randomly selected among 20 different services. The performances of the utility functions and of the composition strategies are evaluated by varying, on the one hand, the maximum number of hops between the service client and service provider from 1 to 3

\footnotetext{
${ }^{1}$ http://casa-irisa.univ-ubs.fr/lepton/
}

while maintaining the number of services per request to 4 , and on the other hand, the number of services per composition from 3 to 6 while keeping the number of hops to 2. Evaluation results are presented in Figure 1.

\section{B. Success ratio of compositions}

The results we obtained show that when the number of hops increases, the success ratio decreases regardless of the strategy and the utility function. This is the result of the additional disruptions occurring between intermediate devices when the number of hops increases. These results also shows that the compositions relying on the location-based utility function (LUF) are less affected than the compositions performed with the time-based utility function (TUF). This can be explained by the fact that closer providers are selected and enrolled in the composition process; disruptions between closer devices occurring more rarely than between devices that are at the limit of their radio range. Moreover, closer mobile devices can meet together and can recover compositions more quickly, thus increasing the success ratio of compositions. It also must be noticed that the success ratio is better in the sport event scenario, because the mobility of people is constrained as they move along the running path.

The composition success ratio decreases when the number of services to compose increases, because the number of failures (or interruptions) in the composition processes, resulting from the mobility of the devices, increases with the number of services. Like in the first evaluations, the configuration that provides the better success ratio is the orchestrationbased strategy with the LUF. The reasons are the same as those related to the variation of the number of hops. In the sport event scenario, the gap between the two strategies and the two utility functions is reduced, and the ratio is better than in the open area scenario, due to the mobility of people being constrained by the running path, as mentioned before. Therefore it is more easy to meet service providers.

\section{Execution time of compositions}

Concerning the execution time of the compositions, the results show that the CS outperforms the OS regardless of the utility function and the scenario. This important gap between the two strategies is mainly due to their running principle, but also to the process of formation of groups in Wi-Fi Direct. Indeed as mentioned previously in this paper, with the OS, the composition request remains on the composition requester, and this one selects and invokes the service providers it has discovered and that must be enlisted in a composition. As shown in [2], with the OS and the communication technology we consider, the services enrolled in a composition are mainly provided by one hop neighbors (i.e., by the Wi-Fi Direct group owners). This difference between the CS and the OS is more significant when the maximum number of hops of messages is limited to one, because the composition requester can only access its local services and those offered by its group owner in its Wi-Fi Direct group. Thus to complete a composition, a composition requester must often leave a group and join another one in order to invoke the rest of services. 

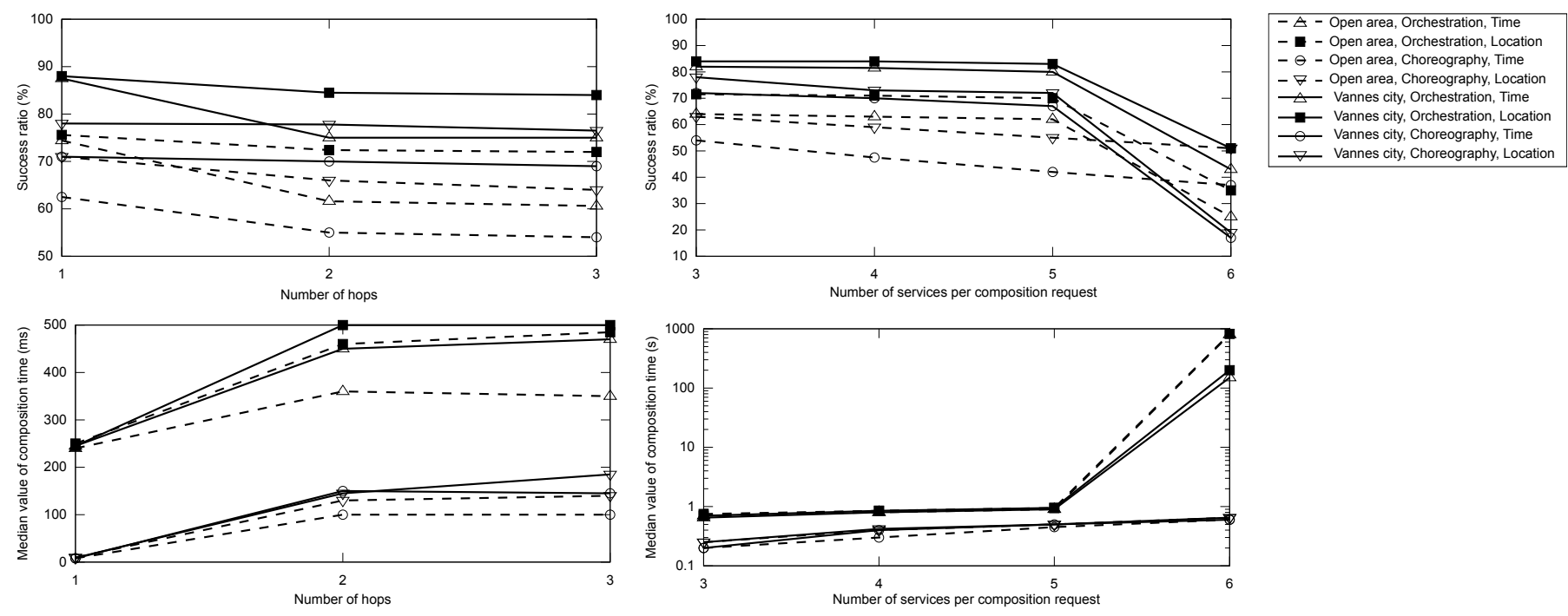

Figure 1. Success ratio and execution time of compositions against the number of hops and the number of services per request.

The CS does not have such limitations, even with a maximum number of hops equal to one, since the composition request is transmitted from one provider to another until the composition is completed. For instance, with a maximum number of hops equal to one, a requester, which is a client of a group owner, can delegate the composition to its group owner, and this one can delegate, in turn, the rest of the composition to one of the providers hosted by its clients. Moreover, with the CS, intermediate responses are not returned to the requester, thus reducing the number of exchanges and the time between two successive invocations of services. The results also show that when the maximum number of hops increases, the execution time of a composition increases too. Indeed, when services to invoke are not available in the current communication group, the requester or the current provider should leave and joins another group where the next service is available. The process of leaving, traveling, and joining another group, introduces additional delays.

The results show that the median of the composition time increases logically and inevitably as the number of services listed in a service composition request increases. This median grows significantly for 6 services per composition. The explanation of this variation resides in the fact that is more difficult to find the sixth service locally or on the close devices, especially for the OS. Indeed, 6 different services are chosen among the 20 existing ones to define the composition requests, knowing that 5 services among the 20 ones are deployed on each devices. The results show that the CS offers better execution times than the OS regardless the number of services per composition and the utility function. The reasons are the same that those detailed in the previous paragraph.

\section{CONCLUSION}

In this paper, we have proposed a service composition system dedicated to opportunistic networks. This system implements two composition strategies and a utility function that relies on two criteria: execution time and success ratio.
Two implementations of this utility function are provided: one relying on time properties, and another one based on location properties.

The evaluation results of the system mainly show that the location information helps to make composition invocations more reliable, but it does not help to select the providers that offer the shortest invocation times. These results also show that the orchestration strategy (OS) guarantees a higher success ratio than the choreography strategy (CS), whereas, from the composition time perspective, the CS proved to be significantly faster than the OS. From these two observations, we argue that it could be relevant to dynamically mix the two strategies in the same composition: the choice of the strategy could be revised at each step of the execution of the composition request, switching from one strategy to the other when needed. Such a dynamic adaptation could reduce the execution time of the compositions, while increasing their success ratio.

\section{REFERENCES}

[1] U. Sadiq, M. Kumar, A. Passarella, and M. Conti, "Service Composition in Opportunistic Networks: A Load and Mobility Aware Solution," IEEE Transactions on Computers, vol. 84, no. 8, pp. 2308-2322, Aug. 2015.

[2] F. Baklouti, N. L. Sommer, and Y. Maheo, "Choreography-based vs orchestration-based service composition in opportunistic networks," in 2017 IEEE 13th International Conference on Wireless and Mobile Computing, Networking and Communications (WiMob), Oct 2017, pp. 1-8.

[3] M. Conti, E. Marzini, D. Mascitti, A. Passarella, and L. Ricci, "Service Selection and Composition in Opportunistic Networks," in 9th International Wireless Communications and Mobile Computing Conference (IWCMC 2013), Cagliari, Italy, July 2013, pp. 1565-1572.

[4] C. Groba and S. Clarke, "Opportunistic Composition of SequentiallyConnected Services in Mobile Computing Environments," in 2011 IEEE International Conference on Web Services, July 2011, pp. 17-24.

[5] S. Kalasapur, M. Kumar, and B. Shirazi, "Seamless Service Composition (SeSCo) in Pervasive Environments," in 1st ACM International Workshop on Multimedia Service Composition (MSC'05). Hilton, Singapore: ACM, 2005, pp. 11-20.

[6] N. Le Sommer, P. Launay, and Y. Mahéo, "A Framework for Opportunistic Networking in Spontaneous and Ephemeral Social Networks," in 10th ACM MobiCom Workshop on Challenged Networks (CHANTS 2015). Paris, France: ACM Press, Sep. 2015, pp. 1-4. 